\title{
Nuevas estrategias en el tratamiento del síndrome antifosfolípido
}

\author{
SEBASTIÁN IBÁÑEZ, MIRENTXU IRURETAGOYENA, \\ MIGUEL A. GUTIÉRREZ
}

\section{New alternatives for the treatment of antiphospholipid syndrome. A literature review}

For years the mainstay of antiphospholipid syndrome treatment has been anticoagulation and antiplatelet therapy, but the autoimmune nature of the disease, and complications of these therapies, created the need to develop new therapeutic strategies. New therapeutic alternatives inhibit at different levels, the cascade of events leading to the pro-thrombotic state characteristic of the antiphospholipid syndrome. We conducted a literature review of these new treatments, focusing on the pathophysiological bases that support them and their possible clinical applications.

(Rev Med Chile 2013; 141: 1041-1048).

Key words: Antiphospholipid syndrome; Hydroxychloroquine, Hydroxymethylglutaryl-CoA Reductase Inhibitors; Therapies, investigational.

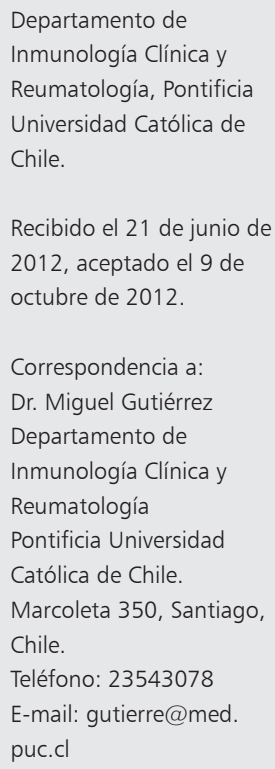

4 1 síndrome antifosfolípido (SAF) es una enfermedad autoinmune caracterizada por trombosis (venosa y/o arterial), y/o pérdidas fetales recurrentes, asociada con la presencia de anticuerpos antifosfolípidos (aPL). La prevalencia de la enfermedad es desconocida, esto debido en parte a múltiples cambios en los criterios diagnósticos. En lupus eritematoso sistémico (LES), 30 a $40 \%$ de los pacientes presentan aPL, y un tercio de estos (10 a 15\% de los pacientes con LES en general) tienen manifestaciones clínicas de $\mathrm{SAF}^{1}$.

Los aPL son autoanticuerpos dirigidos contra epítopes en proteínas plasmáticas, que se generan por la unión de estas proteínas a fosfolípidos. Los tres aPL más importantes son el anticoagulante lúpico, los anticuerpos anti-cardiolipina, y los anticuerpos anti-B2 glicoproteína I (B2GPI) ${ }^{2}$. La B2GPI es un inhibidor natural de la coagulación y de la agregación plaquetaria. Al unirse a fosfolípidos con carga negativa inhibe la activación por contacto de la cascada de la coagulación, y también la conversión de protrombina a trombina. Es el principal blanco de los aPL ${ }^{3}$.

La enfermedad se puede clasificar como SAF primario, o secundario cuando se asocia a otras enfermedades autoinmunes, principalmente LES. El SAF primario es la causa más común de trombofilia adquirida y da cuenta de: 10 a 15\% de todos los episodios de trombosis venosa profunda (TVP), con o sin embolia pulmonar; de un tercio de los nuevos accidentes cerebrovasculares en pacientes menores de 50 años; y de 10 a 15\% de las mujeres con pérdidas fetales recurrentes. La prevalencia estimada de TVP asociada a SAF primario es 0,3 a $1 \%$ de la población general ${ }^{4}$.

Actualmente, no existe tratamiento curativo para el SAF, pero el estado protrombótico provocado por los aPL ha motivado que el pilar del tratamiento sea la anticoagulación y los antiagregantes plaquetarios. A pesar de que los pacientes lleven bien su tratamiento la recurrencia de eventos trombóticos y la presencia de complicaciones obstétricas y hemorrágicas no son raras $^{5}$. El desarrollo de terapias más efectivas y con menos complicaciones es una necesidad actual para este síndrome.

La comprensión de la fisiopatología de la enfermedad ha progresado mucho desde la década 1990-99 gracias a modelos animales (inmunización de ratones con aPL) y el conocimiento 
obtenido de la interacción entre aPL y distintos componentes de la coagulación y del endotelio ${ }^{6}$. Además se ha demostrado que la inflamación es fundamental en el desarrollo de la enfermedad.

Se ha propuesto el siguiente mecanismo de trombosis mediada por aPL (Figura 1): la membrana celular está compuesta por fosfolípidos fosfatidilcolina neutros, y fosfolípidos fosfatidilserina con carga negativa que migran a la capa externa durante la activación o apoptosis de plaquetas y células endoteliales. La B2GPI dimérica normalmente se une a fosfatidilserina por receptores como anexina A2 o receptores tipo Toll (TLR), inhibiendo la activación de la cascada de la coagulación de células endoteliales. Los aPL se unen a B2GPI, interfiriendo con su función y activando el sistema de complemento (C'), induciendo la expresión de C5a que a su vez induce la expresión de moléculas de adhesión (ICAM-1), de citoquinas (IL-1, IL-6, IL-8)y de factor tisular (FT). También se activan monocitos, polimorfonucleares (PMN) y plaquetas, lo que resulta en la liberación de mediadores proinflamatorios, y generación de un estado protrombótico. Además, actualmente se sabe que p38 mitogen activated protein kinase (p38 MAPK) (proteína fundamental para la respuesta celular al estrés) y el factor nuclear $\mathrm{kB}(\mathrm{NF \kappa B})$ (factor de transcripción fundamental en procesos de activación, proliferación, etc.) tienen un rol importante en la cascada de señales intracelulares que llevan a la activación y adhesión de plaquetas, al aumento de expresión de FT y a la liberación de citoquinas ${ }^{7}$.

Gracias al reconocimiento de estos componentes del mecanismo de trombosis en el SAF se han podido elaborar diferentes estrategias para inhibir el efecto de aPL a distintos niveles de acción. Discutiremos el avance de estos estudios y su potencial aplicación clínica. Para esto realizamos una revisión selectiva de las publicaciones indizadas en PubMed, sin carácter de revisión sistemática ni metaanálisis. Se incluyeron trabajos relacionados con la fisiopatología y el tratamiento del SAF publicados en la base de datos Medline.

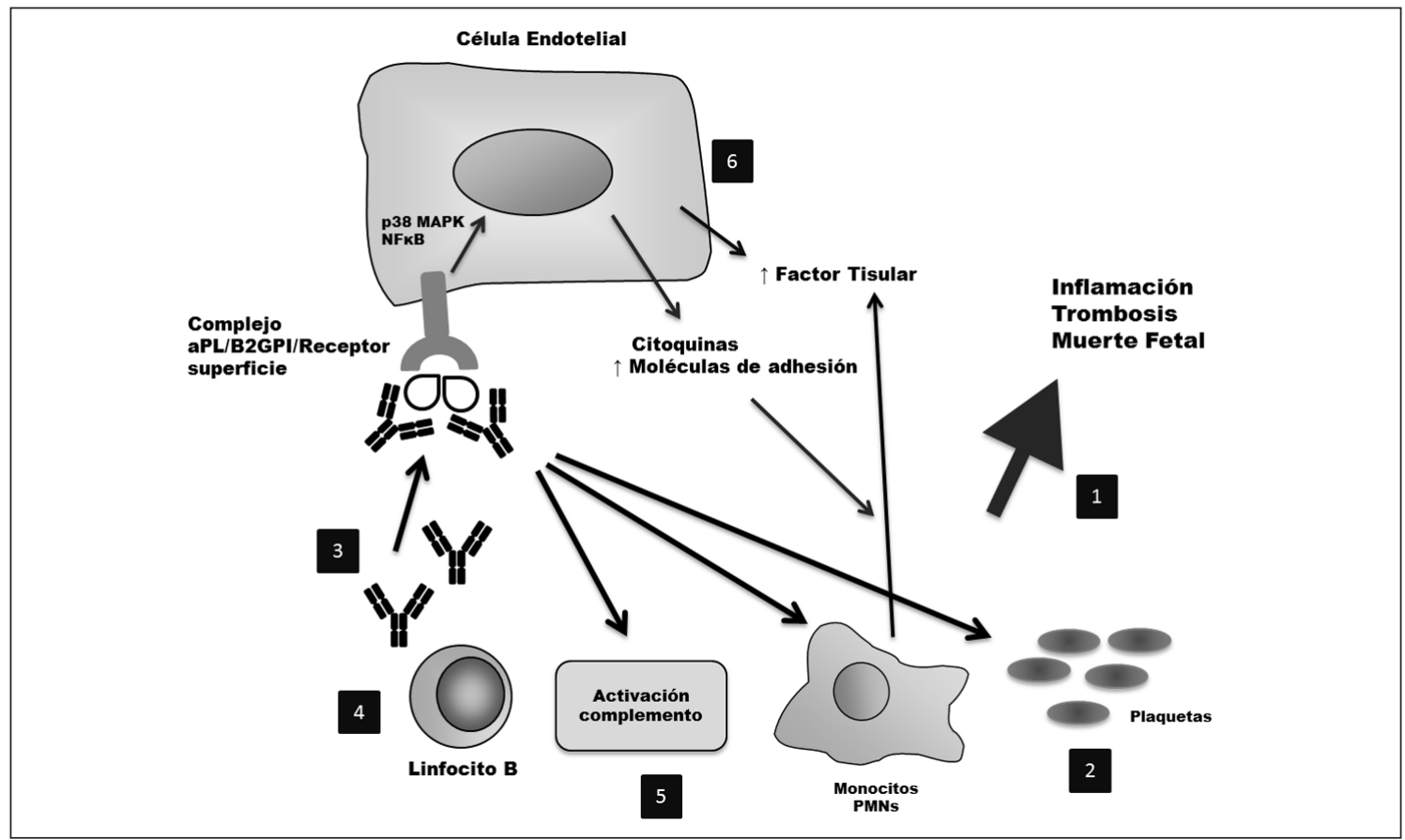

Figura 1. Mecanismos de trombosis mediada por aPL y etapas (números) en las que el efecto de aPL puede ser inhibido. 1) Anticoagulación; 2) Antiagregación plaquetaria; 3) Aumento de clearence de aPL; 4) Inhibición de células B; 5) Inhibición de la activación de complemento inducida por aPL; 6) Inhibición de activación de célula endotelial y/o sobrerregulación de FT inducida por aPL.Abreviaciones: aPL, anticuerpos antifosfolípido; B2GPI, B2-glicoproteina -l; CK, citoquinas; FT, factor tisular; MA, moléculas de adhesión; MN, mononuclear; NFKB, factor nuclear kB; p38MAPK, p38 kinasa activada por mitógeno; PMN, polimorfonuclear; PLT, plaquetas. 


\section{Estatinas}

Estudios sugieren que las estatinas pueden afectar la proliferación y migración del músculo liso, la activación de monocitos, la síntesis de citoquinas y la expresión de $\mathrm{FT}^{8}$.

Primero Meroni y cols. demostraron que las estatinas inhiben la activación endotelial mediada por aPL, al observar una disminución dosis dependiente mediada por fluvastatina de la adhesión de monocitos a células endoteliales de vena umbilical humana (HUVEC) incubadas con LPS, IL-1B, TNF $\alpha$ e IgG antiB2GPI. Fluvastatina también redujo la expresión de selectina E y de moléculas de adhesión intercelular 1 (ICAM-1). El mevalonato suprimió el efecto de la fluvastatina, lo que sugiere que el efecto está mediado por la inhibición de la enzima hidroximetilglutaril coenzima A reductasa (HMG-CoA). Fluvastatina también inhibió la unión de NF- $\kappa$ B al ADN y la expresión de mRNA de IL-6 luego de una estimulación de células HU$\mathrm{VEC}$ con TNF $\alpha$ o IgG antiB2GPI ${ }^{8}$.

Luego Ferrara y cols. demostraron in vivo los efectos de la fluvastatina. Ratas CD-1 fueron tratadas con IgG de pacientes con SAF, con o sin fluvastatina. No hubo diferencias de los niveles de aCL pero fluvastatina disminuyó en forma significativa el tamaño de los trombos, la adhesión de leucocitos al endotelio y los niveles de sICAM- $1^{9}$. Fluvastatina también inhibe el aumento de expresión de FT provocado por aPL en células endoteliales ${ }^{10}$.

Un estudio reciente midió, en el suero de 93 pacientes con SAF y 60 controles, los niveles del factor de crecimiento endotelial vascular (VEGF), FT, TNF $\alpha$, sICAM-1, sE-selectina, PCR, sVCAM-1. Además en 9 pacientes con SAF se midió lo mismo el día de inicio de fluvastatina y a los 30 días de uso (40 mg al día). TNF $\alpha$, FT y VEGF estaban elevados en los pacientes con SAF, y fluvastatina redujo significativamente estos marcadores en la mayoría de los pacientes que fueron tratados ${ }^{11}$.

De manera similar un estudio con 42 pacientes con SAF y 35 sujetos sanos tratados con fluvastatina $20 \mathrm{mg}$ al día por 1 mes mostró reducción significativa de la expresión de FT y VEGF en monocitos, lo que se relacionó con la disminución de la fosforilación de p38 MAPK y de la unión de $\mathrm{NF \kappa B}$ al DNA ${ }^{12}$. Estos resultados sugieren que el uso de estatinas como terapia adyuvante podría ser útil en pacientes con SAF, pero faltan estudios de calidad que evalúen si su uso disminuye los eventos trombóticos en estos pacientes.

\section{Rituximab}

Se ha propuesto que las células B están involucradas en las manifestaciones clínicas del SAF. Estas son necesarias para el inicio de enfermedades relacionadas a autoanticuerpos, como productoras de autoanticuerpos, moduladoras de señales y presentadoras de autoantígenos ${ }^{13}$.

Kahn y cols. demostraron que al bloquear el factor de supervivencia de células B (BAFF) en un modelo animal, mediante inmunoglobulinas que bloquean su receptor, se puede prevenir algunas manifestaciones de la enfermedad, retardar el inicio de la enfermedad y prolongar la sobrevida. En los ratones tratados, los bazos eran más pequeños, con menos células B y T, activadas y de memoria. No previno el desarrollo de anticuerpos anticardiolipina, y sólo produjo retraso modesto de trombocitopenia, pero sí hubo significativamente menos nefritis e infartos miocárdicos ${ }^{14}$. Este estudio apoyó la creencia que las células B juegan un rol importante en la patogénesis de la enfermedad.

Existen casos aislados reportados de uso exitoso de rituximab, principalmente en SAF catastrófico ${ }^{15}$. En el estudio RITAPS se trataron con rituximab 20 pacientes con manifestaciones resistentes a anticoagulación. A fines de 2012 se esperan resultados. El outcome primario es seguridad ${ }^{16}$.

Todavía no existe suficiente evidencia para recomendar rituximab en SAF, pero pronto podríamos tener resultados que nos permitirán sustentar una recomendación.

\section{Hidroxicloroquina (HCQ)}

La HCQ fue utilizada en las décadas 1970-79 y 1980-89 como profilaxis de trombosis venosa profunda en postoperatorio de cirugías ortopédicas por su capacidad para disminuir la viscosidad sanguínea y la agregación plaquetaria ${ }^{17,18}$. Se reportaron resultados negativos, principalmente en cirugía de cadera, y finalmente se dejó de utilizar ${ }^{19,20}$. En la década 1990-99 renace el interés por HCQ, con la publicación de un estudio, en un modelo animal de SAF, donde la HCQ redujo el tamaño del trombo. Este efecto fue dosis dependiente ${ }^{21}$. 
En el año 2002 se publicó un estudio donde se evaluó un grupo de pacientes con SAF (con eventos trombóticos) y pacientes con LES portadores asintomáticos de aPL. Mediante un análisis de regresión logística se demostró que si los pacientes tomaban HCQ o aspirina disminuía la probabilidad de presentar un evento trombótico ${ }^{22}$.

Rand y cols. demostraron en el 2008, mediante elipsometría y microscopia de fuerza atómica (AFM), que la HCQ inhibía directamente la formación de complejos inmunes IgGaPL-B2GPI en la bicapa de fosfolípidos y disminuía significativamente los niveles de aPL, en forma dosis dependiente ${ }^{23}$.

Recientemente, se publicó un nuevo efecto de HCQ, sobre anexina A5 (AnxA5). AnxA5 es una potente proteína anticoagulante que se cristaliza sobre la bicapa de fosfolípidos bloqueando su disponibilidad para reacciones de coagulación. La unión de AnxA5 a la bicapa se altera por aPL, favoreciendo la aparición de trombosis y abortos. HCQ revirtió el efecto de los aPL, aumentando la actividad anticoagulante de AnxA5 en plasma de pacientes con $\mathrm{SAF}^{24}$. En el año 2011 se publicó evidencia de este mismo efecto en el sincitiotrofoblasto, donde la HCQ redujo la unión de aPL al sincitiotrofoblasto y restauró la expresión de AnxA5 ${ }^{25}$.

En el ámbito clínico sólo existe evidencia de calidad moderada de efecto benéfico de HCQ sobre trombosis en pacientes con LES, resultados no necesariamente aplicables a pacientes con $\mathrm{SAF}^{26}$.

A pesar de esto, y dado la evidencia del efecto de HCQ sobre los procesos trombogénicos, y su buen perfil de seguridad, parece razonable recomendar su uso para prevenir trombosis en pacientes con SAF.

\section{Inhibición de la sobrerregulación de factor tisular (FT)}

En 1997 se publicó uno de los primeros estudios en pacientes sobre el rol del FT en SAF. En pacientes con SAF, con y sin trombosis y en controles sin SAF, con y sin trombosis, se midió la expresión de FT en monocitos. Esta fue mayor en el grupo con SAF y trombosis. Esto se relacionaba con la presencia de anticuerpos IgG anticardiolipina ${ }^{27,28}$.

Dado que los monocitos sintetizan FT y contienen varios componentes del sistema renina angiotensina se investigó un posible efecto de inhibidores de enzima convertidora de angiotensina (IECAs) sobre la expresión de FT. Cultivaron monocitos de pacientes sanos con endotoxina en presencia de diferentes IECAs. Captopril redujo la expresión de FT en la membrana en $60 \%$ aproximadamente luego del estímulo con endotoxina. Otros IECAs y losartán tuvieron el mismo efecto. El aumento de niveles de mRNA de FT, mediado por endotoxinas, fue inhibido por captopril, junto con la translocación de NF- $\kappa \mathrm{B}$ al núcleo y su unión a la región promotora del gen de $\mathrm{FT}^{29}$.

Para investigar el efecto en SAF de dilazep y dipiridamol, ambos antiagregantes plaquetarios, cultivos primarios de monocitos fueron estimulados con LPS, IgG SAF, e IgG de paciente sanos, y se observó un aumento de actividad de FT y de la expresión de mRNA de FT en los 2 primeros casos. Esto fue inhibido por dilazep y dipiridamol, de forma dosis dependiente. No tienen efecto sobre el aumento de niveles de mRNA de FT, a diferencia de captopril, por lo que el bloqueo de la expresión de FT es post transcripcional ${ }^{30}$.

Para poder entregar una recomendación mejor sustentada sobre el uso de estos agentes en pacientes con SAF se requieren estudios in vivo.

\section{Inhibición de complemento}

La activación de complemento parece ser requisito integral para la activación de elementos celulares blancos de aPL. Esto fue demostrado por un estudio de Fischetti y cols., quienes purificaron IgG aPL de pacientes con SAF, e inyectaron LPS intraperitoneal a ratas, $3 \mathrm{~h}$ antes de tratarlas con las IgG purificadas. Esto indujo la formación de trombos, lo que no sucedió si las IgG purificadas eran depletadas de anti-B2GPI. Cuando se repitió el experimento en ratas deficientes en C6 no se formaron trombos. Al inhibir la función de C5 también se inhibió la trombosis, por lo tanto, se concluyó que la presencia de C5 y C6 es necesaria para la formación de trombos luego de la estimulación con $\operatorname{IgG} \mathrm{aPL}^{31}$. Además, se demostró que la activación de C3 también es necesaria para la trombofilia mediada por aPL al comparar el efecto de IgG de SAF en ratas wild type (WT) y en ratas deficientes de $\mathrm{C} 3$ y C5, las que formaron trombos de menor tamaño ${ }^{32}$.

Estos estudios demuestran el importante rol 
del complemento en el SAF, y es un área de investigación interesante para el desarrollo de terapias futuras.

\section{Antagonistas del TNF- $\alpha$}

Berman y cols. evaluaron el rol de TNF $\alpha$ en complicaciones del embarazo provocadas por aPL en un modelo animal de SAF. Mostraron que aPL tienen como blanco la decidua y que causan un rápido aumento de los niveles locales y sistémicos de TNF $\alpha$. Las ratas deficientes en TNF $\alpha$, o en las que se bloqueó este factor, presentaron menos pérdidas fetales. También observaron que en ratas deficientes en C5 no aumentaron los niveles de TNF $\alpha$ al tratarlas con aPL. Esto sugirió que TNF $\alpha$ tiene rol patogénico en $\mathrm{SAF}^{33}$.

Blanky cols. vacunaron ADN codificante de TNF $\alpha$ a ratas con SAF experimental inducido por B2GPI. Esto resultó en la generación de memoria inmunológica para el producto génico de la vacuna, con aumento de anticuerpos anti TNF $\alpha$ circulantes. La fracción enriquecida de $\operatorname{IgG}$ de anticuerpos antiTNF $\alpha$ de estos ratones fue biológicamente activa, ya que previno la activación de células endoteliales por TNF $\alpha$, de manera similar a anti-TNF $\alpha$ comercial, medido por el porcentaje de inhibición de adhesión de monocitos. Ratas inmunizadas con B2GPI, vacunadas con ADN de TNF $\alpha$ en etapa temprana de la enfermedad, mostraron disminución de títulos de anticuerpos antiB2GPI circulantes. Esto se siguió de una disminución de las pérdidas fetales, aumento de recuento de plaquetas y normalización de TTPK. Cuando la exposición a la vacuna fue tardía el efecto fue menor ${ }^{34}$.

No existen reportes de uso de terapia anti-TNF en SAF, si de casos de posible elevación de aCL por su uso. Se requiere de mayores estudios para realizar una recomendación.

\section{Inhibidores de p38 MAPK y NF- $\kappa \mathrm{B}$}

La quinasa p38 MAPK es clave en la vía intracelular de activación de plaquetas, células endoteliales y monocitos por aPL, y NF- $\kappa \mathrm{B}$ es parte de la cascada de transducción intracelular que lleva al aumento de FT en el endotelio y monocitos.
Vega-Ostertag demostró que en plaquetas tratadas con IgG aPL y dosis subactivantes de trombina aumenta significativamente la fosforilación de p38 MAPK. Al utilizar un inhibidor específico de p38 MAPK se interfiere la agregación plaquetaria y la expresión de $\mathrm{FT}^{35,36}$.

Existen reportes de uso de inhibidores de p38 MAPK en otros modelos de enfermedad como isquemia cerebral, shock séptico, artritis e isquemia miocárdica, y actualmente se están estudiando nuevos inhibidores ${ }^{37,38}$.

Otros autores han demostrado que inhibir la función de NF- $\kappa \mathrm{B}$ interfiere con la trombosis inducida por $\mathrm{aPL}^{39,40}$.

$\mathrm{Al}$ bloquear la cascada intracelular inducida por aPL mediante inhibidores de NF- $\kappa$ B y de p 38 MAPK se obtiene resultados antitrombóticos en modelos animales, pero no existen datos de eficacia y seguridad en humanos.

\section{Bloqueo de receptores en células blanco}

Al bloquear receptores de B2GPI o aPL en células blanco se pararía la cascada que lleva a trombosis.

Parece ser que anexina A2 es receptor de B2GPI. Ma y cols. demostraron que los Ac anti anexina A2 bloquean la unión de B2GPI a células endoteliales ${ }^{41}$. TLR4 es el receptor de LPS, que activa la fosforilación de p38 MAPK y la translocación de NF- $\kappa \mathrm{B}$ al núcleo. Mutaciones en el gen codificante de TLR4 interfieren con la respuesta a LPS. Pierangeli demostró que ratas con esta mutación tenían trombos más pequeños y menos adhesión de leucocitos a CE. Además, en pacientes con SAF la prevalencia de polimorfismos protectores (con mutación de TLR) fue menor que en controles ${ }^{42}$.

Un péptido sintético de 20 aminoácidos que comparte similitudes con el dominio V de B2GPI, TIFI, mostró inhibir el estado protrombótico inducido por aPL en ratones, al competir con B2GPI para unirse a la membrana fosfolipídica de la célula blanco ${ }^{43}$. Distintos péptidos similares han sido evaluados en modelos animales demostrando disminución del daño ${ }^{44}$.

Con estos datos, el bloquear la unión de aPL a sus células blanco por competencia con péptidos parece ser una atractiva forma de tratar el SAF, que requiere mayor investigación. 
Tabla 1. Resumen de tratamientos propuestos

\begin{tabular}{|c|c|c|}
\hline Tratamiento & Efecto observado & Estudios publicados \\
\hline Estatinas & $\begin{array}{l}\text { Inhibición de FT y de trombosis induci- } \\
\text { da por aPL }\end{array}$ & $\begin{array}{l}\text { In vitro y modelos murinos. Estudios } \\
\text { piloto en pacientes }\end{array}$ \\
\hline Rituximab & Mejoría clínica & Reportes de casos en SAF y LES \\
\hline Hidroxicloroquina & $\begin{array}{l}\text { Inhibición de formación de complejos } \\
\text { inmunes, del aumento de la activa- } \\
\text { ción plaquetaria y de la disrupción de } \\
\text { anexina A5 mediada por aPL }\end{array}$ & $\begin{array}{l}\text { In vitro y modelos murinos. Estudios } \\
\text { en pacientes con LES }\end{array}$ \\
\hline Dilazep y dipiridamol & Inhibición de FT & In vitro \\
\hline IECA & Inhibición de sobrerregulación de FT & In vitro \\
\hline Inhibidores de complemento & $\begin{array}{l}\text { Disminución de abortos, trombosis } \\
\text { y activación de células endoteliales } \\
\text { mediado por aPL }\end{array}$ & In vitro y modelos murinos \\
\hline Péptidos que imitan regiones de B2GPI & $\begin{array}{l}\text { Bloquean la unión de B2GPI/aPL a } \\
\text { células blanco }\end{array}$ & In vitro y modelos murinos \\
\hline Inhibidores de p38 MAPK y NF-кB & $\begin{array}{l}\text { Interfieren agregación plaquetaria y } \\
\text { expresión de FT }\end{array}$ & In vitro y modelos murinos \\
\hline Antagonistas de TNFa & $\begin{array}{l}\text { Inhibe la activación de células endote- } \\
\text { liales y disminución de aPL }\end{array}$ & In vitro y modelos murinos \\
\hline
\end{tabular}

\section{Glucocorticoides}

Aunque el uso de glucocorticoides en el SAF es antiguo, con resultados variables y alta frecuencia de complicaciones, la controversia aún continúa. Recientemente se publicó el uso de prednisolona en dosis baja en pacientes con SAF obstétrico refractario. Dieciocho mujeres con diagnóstico de SAF y al menos un aborto previo a pesar del tratamiento con aspirina y heparina, se trataron con prednisolona $10 \mathrm{mg}$ además de aspirina y heparina de bajo peso molecular. En los embarazos previos de estas mujeres sólo 4 de 97 (4\%) resultaron en recién nacidos vivos. De 23 embarazos suplementados con prednisolona, 9 mujeres tuvieron 14 recién nacidos vivos (61\%), 8 sin complicaciones ${ }^{45}$. Como es el único estudio que ha mostrado resultados positivos con esta asociación de tratamiento, se necesita mayor investigación.

\section{Conclusiones}

La naturaleza multifactorial de la trombosis en el síndrome antifosfolípido es una barrera importante para el desarrollo de nuevas terapias.
En los últimos años, la comprensión de los mecanismos de trombosis mediada por aPL ha establecido el importante rol de la inflamación, lo que puede hacer posible que en el futuro el enfrentamiento antitrombótico actual sea complementado por un tratamiento inmunomodulatorio.

Es probable que las estatinas, hidroxicloroquina, Rituximab, IECAs, e incluso drogas anti-TNF sean parte del tratamiento estándar de SAF en el futuro y que continúen las investigaciones en otras áreas prometedoras como inhibidores de p38MA$\mathrm{PK}$, de NF- $\kappa \mathrm{B}$, de complemento, y bloqueadores de receptores en células blanco (Tabla 1).

Son necesarios ensayos clínicos bien diseñados para validar la utilidad de estos agentes.

\section{Referencias}

1. Levine JS, Branch DW, Rauch J. The antiphospholipid syndrome. N Engl J Med 2002; 346: 752-63.

2. Roubey RA. Immunology of the antiphospholipid antibody syndrome. Arthritis Rheum 1996; 39 (9): 1444.

3. Ichikawa K, Khamashta MA, Koike T, Matsuura E, Hughes GR. beta 2-Glycoprotein I reactivity of monoclonal anticardiolipin antibodies from patients with the 
antiphospholipid syndrome. Arthritis Rheum 1994; 37 (10): 1453.

4. Ginsburg KS, Liang MH, Newcomer L, et al. Anticardiolipin antibodies and the risk for ischemic stroke and venous thrombosis. Ann Intern Med 1992; 117: 997-1002.

5. Ruiz-Irastorza G, Khamashta MA, Hunt BJ, Escudero A, Cuadrado MJ, Hughes GR. Bleeding and recurrent thrombosis in definite antiphospholipid syndrome: analysis of a series of 66 patients treated with oral anticoagulation to a target international normalized ratio of 3.5. Arch Intern Med 2002; 162 (10): 1164.

6. Gharavi AE, Aron AL. Experimental models for antiphospholipid studies. Haemostasis 1994; 24: 204-7.

7. Erkan D, Lockshin MD. New approaches for managing antiphospholipid syndrome. Nat Clin Pract Rheumatol 2009; 5: 160-70.

8. Meroni PL, Raschi E, Testoni C, Tincani A, Balestrieri G, Molteni R, et al. Statins prevent endothelial cell activation induced by antiphospholipid (anti-beta2glycoprotein I) antibodies: effect on the proadhesive and proinflammatory phenotype. Arthritis Rheum 2001; 44: 2870-8.

9. Ferrara DE, Liu X, Espinola RG, Meroni PL, Abukhalaf I, Harris EN, et al. Inhibition of the thrombogenic and inflammatory properties ofantiphospholipid antibodies by fluvastatin in an in vivo animalmodel. Arthritis Rheum 2003; 48: 3272-9.

10. Ferrara DE, Swerlick R, Casper K, Meroni PL, VegaOstertag ME, Harris EN, et al. Fluvastatin inhibits upregulation of tissue factor expression by antiphospholipid antibodies on endothelial cells. J Thromb Haemost 2004; 2: 1558-63.

11. Jajoria P, Murthy V, Papalardo E, Romay-Penabad Z, Gleason C, Pierangeli SS. Statins for the treatment of antiphospholipid syndrome? Ann N Y AcadSci 2009; 1173: 736-45

12. López-Pedrera C, Ruiz-Limón P, Aguirre MÁ, Barbarroja N, Pérez-Sánchez C, Buendía P, et al. Global effects of fluvastatin on the prothrombotic status of patients with antiphospholipid syndrome. Ann Rheum Dis 2011 Apr; 70 (4): 675-82.

13. Youinou P, Renaudineau Y. The antiphospholipid syndrome as a model for B cell-induced autoimmune diseases. Thromb Res 2004; 114: 363-9.

14. Kahn P, Ramanujam M, Bethunaickan R, Huang W, Tao $\mathrm{H}$, Madaio MP, et al. Prevention of murine antiphospholipid syndrome by BAFF blockade. Arthritis Rheum 2008; 58: 2824-34.

15. Iglesias-Jiménez E, Camacho-Lovillo M, FalcónNeyra D, Lirola-Cruz J, Neth O. Infant with probable catastrophic antiphospholipid syndrome successfully managed with rituximab. Pediatrics 2010; 125 (6): e1523-8.

16. A Pilot Study of Rituximab for the Anticoagulation Resistant Manifestations of Antiphospholipid Syndrome (RITAPS). [http://clinicaltrials.gov/ct2/ show/NCT0053 7290?term=NCT00537290\&rank=1].

17. Carter AE, Eban R. Prevention of postoperative deep venous thrombosis in legs by orally administered hydroxychloroquine sulphate. Br Med J 1974; 3: 94-5.

18. Chrisman DO, Snook GA, Wilson TC, Short JY. Prevention ofvenous thromboembolism by administration of hydroxychloroquine. J Bone Joint Surg 1976; 58A: 91820.

19. Cooke ED, Dawson MHO, Ibbotson RM, et al. Failure of orally administered hydroxychloroquine sulphate to prevent venous thromboembolism following elective hip operations. J Bone Joint Surg 1977; 59A: 496-500.

20. Loudon JR: Hydroxychloroquine and postoperative thromboembolism after total hip replacement. Am J Med 1988; 85 (Suppl4A): 57-61.

21. Edwards MH, Pierangeli S, Liu X, Barker JH, Anderson G, Harris EN. Hydroxychloroquine reverses thrombogenic properties of antiphospholipid antibodies in mice. Circulation 1997; 96: 4380-4.

22. Erkan D, Yazici Y, Peterson MG, et al. A cross-sectional study of clinical thrombotic risk factors and preventive treatments in antiphospholipid syndrome. Rheumatology (Oxford) 2002, 41: 924-9.

23. Rand JH, Wu XX, Quinn AS, Chen PP, Hathcock JJ, Taatjes DJ. Hydroxychloroquine directly reduces the binding of antiphospholipid antibody-beta2-glycoprotein I complexes to phospholipid bilayers. Blood 2008; 112: 1687-95.

24. Rand JH, Wu XX, Quinn AS, Ashton AW, Chen PP, Hathcock JJ, et al. Hydroxychloroquine protects the annexin A5 anticoagulant shield from disruption by antiphospholipid antibodies: evidence for a novel effect for an old antimalarial drug. Blood 2010; 115 (11): 2292-9. Epub 2009 Nov 30.

25. Xiao-Xuan Wu MD, Seth Guller PhD, Jacob H Rand M. Hydroxychloroquine reduces binding of antiphospholipid antibodies to syncytiotrophoblasts andrestores annexin A5 expression. Am J Obstet Gynecol 2011; 205: 576. e7-14.

26. Ruiz-Irastorza G, Ramos-Casals M, Brito-Zeron P, Khamashta MA.Clinical efficacy and side effects of antimalarials in systemic lupus erythematosus: a systematic review. Ann Rheum Dis 2010; 69: 20-8.

27. Kinev AV, Roubey RA. Tissue factor in the antiphospholipid syndrome. Lupus 2008; 17 (10): 952-8.

28. Cuadrado MJ, López-Pedrera C, Khamashta MA, Camps 
MT, Tinahones F, Torres A, et al. Thrombosis in primary antiphospholipid syndrome: a pivotal role for monocyte tissue factor expression. Arthritis Rheum 1997; 40 (5): 834-41.

29. Napoleone E, Di Santo A, Camera M, Tremoli E, Lorenzet R. Angiotensin-converting enzyme inhibitors downregulate tissue factor synthesis in monocytes. Circ Res 2000; 86 (2): 139-43.

30. Zhou H. Dilazep and dipyridamole inhibit tissue factor expression on monocytes induced by IgG from patients with antiphospholipid syndrome. Acta Pharmacol Sin 2004; 25: 1366-71.

31. Fischetti F, Durigutto P, Pellis V, Debeus A, Macor P, Bulla $\mathrm{R}$, et al. Thrombus formation induced byantibodies to beta2-glycoprotein I is complement dependent and requires a primingfactor. Blood 2005; 106 (7): 2340-6. Epub 2005 Jun 14.

32. Pierangeli SS, Girardi G, Vega-Ostertag M, Liu X, Espinola RG, Salmon J. Requirement of activation of complement C3 and C5 for antiphospholipid antibody-mediated thrombophilia. Arthritis Rheum 2005; 52: 2120-4.

33. Berman J, Girardi G, Salmon JE. TNF-alpha is a critical effector anda target for therapy in antiphospholipid antibody-induced pregnancy loss. J Immunol 2005; 174: 485-9.

34. Blank M, Krause I, Wildbaum G, Karin N, Shoenfeld Y. TNFalpha DNA vaccination prevents clinical manifestations of experimental antiphospholipid syndrome. Lupus 2003; 12 (7): 546-9.

35. Vega-Ostertag M, Harris EN, Pierangeli SS. Intracellular events inplatelet activation induced by antiphospholipid antibodies in the presence of low doses of thrombin. Arthritis Rheum 2004; 50: 2911-9.

36. Vega-Ostertag M, Casper K, Swerlick R, Ferrara D, Harris EN, Pierangeli SS. Involvement of p38 MAPK in the up-regulation of tissue factor on endothelial cells by antiphospholipid antibodies. Arthritis Rheum 2005; 52: 1545-54.

37. Barone FC, Irving EA, Ray AM, Lee JC, Kassis S, Kumar S, et al. SB 239063, a second-generation p38 mitogen- activated protein kinase inhibitor, reduces brain injury and neurological deficits in cerebral focal ischemia. J Pharmacol ExpTher 2001; 296: 312-21.

38. Branger J, van den Blink B, Weijer S, Gupta A, van Deventer SJ, Hack CE, et al. Inhibition of coagulation, fibrinolysis, and endothelial cell activation by a p38 mitogen-activated protein kinase inhibitor during human endotoxemia. Blood 2003; 101: 4446-8.

39. Montiel-Manzano G, Romay-Penabad Z, Papalardo de Martínez E, Meillon-García LA, García-Latorre E, Reyes-Maldonado E, et al. In vivo effects of an inhibitor of nuclear factor-kappa B on thrombogenic properties ofantiphospholipid antibodies. Ann N Y Acad Sci 2007; 1108: 540-53.

40. Kubota T, Fukuya Y, Hashimoto R, Kanda T, Suzuki H, Okamura Y, et al. Possible involvement of chemokineinduced platelet activation in thrombophilic diathesis of antiphospholipid syndrome. Ann N Y AcadSci 2009; 1173: 137-45.

41. Ma K, Simantov R, Zhang JC, Silverstein R, Hajjar KA, McCrae KR. High affinity binding of beta 2-glycoprotein I to human endothelial cells is mediated by annexin II. J Biol Chem 2000; 275: 15541-8.

42. Pierangeli SS, Vega-Ostertag ME, Raschi E, Liu X, Romay-Penabad Z, De Micheli V, et al. Toll-like receptor and antiphospholipid mediated thrombosis: in vivo studies. Ann Rheum Dis 2007; 66: 1327-33.

43. Ostertag MV, Liu X, Henderson V, Pierangeli SS. A peptide that mimics the $\mathrm{Vth}$ region of beta-2-glycoprotein I reverses antiphospholipid-mediated thrombosis in mice. Lupus 2006; 15: 358-65.

44. Fleming SD, Pope MR, Hoffman SM, Moses T, Bukovnik U, Tomich JM, et al. Domain V peptides inhibit beta2-glycoprotein I-mediated mesentericischemia/ reperfusion-induced tissue damage and inflammation. J Immunol 2010; 185 (10): 6168-78.

45. Bramham K, Thomas M, Nelson-Piercy, Khamashta M, Hunt B. First trimester low dose prednisolone in refractory antiphospholipid antibody related pregnancy loss. Blood 2011; 117: 6948-51. 